\title{
Pneumatic smart surfaces with rapidly switchable dominant and latent superhydrophobicity
}

\author{
Jian-Nan Wang ${ }^{1}$, Yu-Qing Liu ${ }^{1}$, Yong-Lai Zhang ${ }^{1}$, Jing Feng ${ }^{1}$ and Hong-Bo Sun ${ }^{1,2}$ \\ Smart surfaces that possess switchable wettability are highly desired for a broad range of applications. However, the realization \\ of novel approaches enabling complete alteration of surface properties independent of chemical environment and special \\ materials is still challenging. Herein, inspired by the air sacs of insects, we fabricate a pneumatic smart surface that possesses \\ dual-property wetting behavior and permits fast switching between states. The pneumatic surface is based on an embedded \\ micro-air-sac network composed of an elastomer that was fabricated via a stretching-assisted mismatch-bonding process. By \\ simply pumping the air sacs, the surface could undergo rapid and large-amplitude topography deformation, thereby exposing one \\ surface and hiding the other, and the dominant surface and the latent surface could be switched reversibly. As a typical \\ example, we demonstrate a smart surface with contrasting 'petal' and 'lotus' effects that enables the on-demand capture and \\ release of water droplets. Our pneumatic strategy demonstrates a currently underexploited platform for the development of
} switchable smart surfaces.

NPG Asia Materials (2018) 10, e470; doi:10.1038/am.2017.218; published online 16 February 2018

\section{INTRODUCTION}

With the aid of superwettable biointerfaces, ${ }^{1}$ natural creatures have developed a myriad of survival skills, for instance, self-cleaning, ${ }^{2}$ aquatic walking ${ }^{3}$ and fog collection, ${ }^{4}$ that inspire the development of artificial surfaces with superb wetting properties that are similar or superior to those observed in nature. ${ }^{5-7}$ For example, derived from the lotus effect, ${ }^{8}$ superhydrophobic surfaces with low water adhesion can promote rapid droplet departure, enabling a broad range of applications, such as corrosion resistance, ${ }^{9}$ drag reduction ${ }^{10}$ and anti-icing. ${ }^{11}$ In contrast, the adhesive superhydrophobic surfaces recently observed on rose petals ${ }^{12}$ can facilitate droplet pinning, and they exhibit great potential for droplet manipulation ${ }^{13}$ and nanopatterning. ${ }^{14}$ As each wetting mode features different properties and functions, solid surfaces with a fixed wetting behavior are significantly limited in some cuttingedge applications, the most obvious example of which is the development of intelligent devices, ${ }^{15-20}$ in which there is a need to control the surface wettability dynamically and reversibly. ${ }^{21-23}$ Hence, smart surfaces that possess multiple dewetting properties and permit flexible switching between them, similar to chameleons that expertly change camouflage colors on demand, are highly desired.

It has been well established that surface wettability is governed by chemical composition and geometric structure. To acquire tunable surface wettability, research efforts have been substantially devoted to attaining exquisite control over surface chemistry. By building hierarchical micro-/ nanostructures with stimuli-responsive materials, researchers have successfully prepared various smart surfaces with switchable wettability. ${ }^{6,22-26}$
However, these actuations primarily originate from a gradual change in surface energy that generally requires a long response time. On the other hand, surface structure reconfiguration is an appealing alternative to realize dynamic wettability control. Typically, switchable wetting behaviors have been achieved by bending a soft substrate to a large degree ${ }^{27}$ and by fabricating recoverable structures with shape memory polymers. ${ }^{19}$ Although these pioneering works have provided helpful insights into the controllable switching of surface dewetting properties, there is still a lack of efficient and secure strategies that can realize surface switching on demand. Currently, the realization of new strategies that enable the complete alteration of surface wetting properties independent of chemical environment and special materials is still promising but challenging.

In nature, living organisms have orchestrated biological actuation via volumetric changes to facilitate biological activities. Many insects have evolved air sacs and ramifications in the thorax and abdomen to help them breathe, ventilate, maintain external form and improve blood circulation via expansion/shrinkage modulation. ${ }^{28}$ Inspired by the air sacs of natural creatures, pneumatic strategies have been widely adopted in various automatic systems, such as soft minirobots, ${ }^{29,30}$ tunable microlenses ${ }^{31}$ and microfluidic valves. ${ }^{32}$ However, pneumatic actuation has not yet been employed for wettability modulation as it requires more refined topological control at the micro-/nanoscale to access elaborate wetting states. Here, by integrating a pneumatic micro-air-sac system, we design and fabricate a novel smart surface that possesses dual characters and permits facile switching between them. Selective modification of the micro air sacs with different

\footnotetext{
${ }^{1}$ State Key Lab of Integrated Optoelectronics, College of Electronic Science and Engineering, Jilin University, Changchun, China and ${ }^{2}$ State Key Lab of Precision Measurement technology and Instruments, Department of Precision Instrument, Tsinghua University, Beijing, China Correspondence: Professor Y-L Zhang, State Key Lab of Integrated Optoelectronic, Jilin University, 2699 Qianjin Street, Gaoxin, Changchun, Jilin 130012, China. E-mail: yonglaizhang@jlu.edu.cn or Professor H-B Sun, State Key Lab of Precision Measurement technology and Instruments, Department of Precision Instrument, Tsinghua University, Haidian, Beijing 100084, China. 
materials or nanostructures endows the surface with distinct dominant/latent properties: its wetting state can be reversibly switched by exposing or hiding the micro air sacs through pneumatic control. As an example, we realized rapid and reversible switching between a 'petal effect' and a 'lotus effect' under ambient conditions. Owing to the transition between the 'pinned' and 'roll-down' ultrahydrophobic states, we further developed a smart actuator that allowed the capture and release of water droplets on demand.

\section{MATERIALS AND METHODS}

\section{Fabrication of the pneumatic surfaces}

Supplementary Figure S1a shows the fabrication process of the pneumatic surfaces. First, glass slides were cleaned with acetone, absolute ethanol and deionized water. A layer (ca. $150 \mu \mathrm{m}$ ) of photoresist (SU-8 2025, MicroChem Corporation, Westborough, MA, USA) was spin-coated onto the glass slides. By photolithography, a series of groove arrays arranged in a leaf shape were obtained with different grating periods $(300,400$ and $500 \mu \mathrm{m})$. Second, a mixture of polydimethylsiloxane (PDMS) base and curing agent (Sylgard 184 silicone elastomer kit, Dow Corning Corporation, Auburn, MI, USA) (10:1 by weight) was poured into the photoresist molds and cured at $85^{\circ} \mathrm{C}$ for $2 \mathrm{~h}$. The peeled-off PDMS replicas were used as elastomer substrates. Meanwhile, thin elastomer slices $(\mathrm{ca} .40 \mu \mathrm{m})$ were also prepared on glass slides by spin-coating using the same PDMS mixture. Third, the extendable substrate was pre-stretched on a homemade stage, whereas the cover slice was kept at the original length. Both of them were treated with $\mathrm{O}_{2}$ plasma for $1 \mathrm{~min}$ and were brought into contact to form covalent bonds. Relaxation of the substrate deflected the cover slice, forming protuberant micro air sacs. By tuning the level of strain, a series of micro air sacs were prepared with different heights ranging from 40 to $100 \mu \mathrm{m}$. To further construct a nanostructure on the pneumatic surface, the pneumatic surface was reversed and held in a candle flame for $2 \mathrm{~s}$, allowing carbon soot to be uniformly deposited on the entire surface. ${ }^{33}$ Then, a piece of scotch tape was applied to selectively remove the top layer of the coating (ca. $12 \mu \mathrm{m}$ ) from the inflated surface in order to obtain a hybrid structure on the surface.

\section{External pumping system}

To connect the micro-pneumatic network with the off-board gas source, Teflon tubing with an outer diameter of $1.8 \mathrm{~mm}$ was used. A thick PDMS stamp (ca. $4 \mathrm{~mm}$ ) was bonded on the thin cover slice to fasten the tubing with a drilled
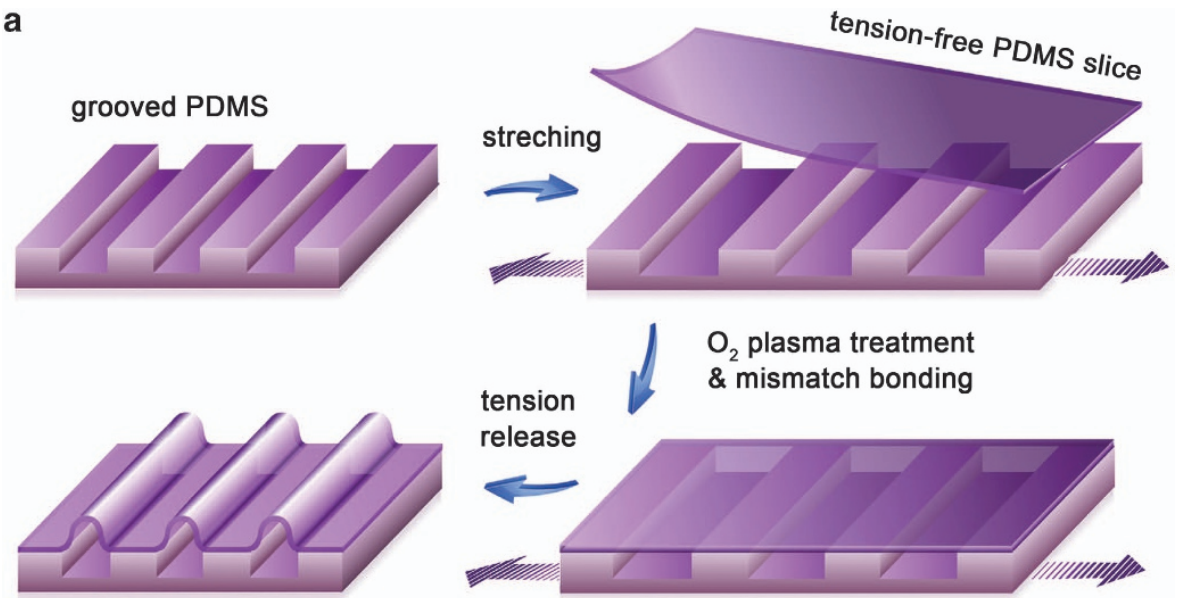

selective deposition or selective structuring

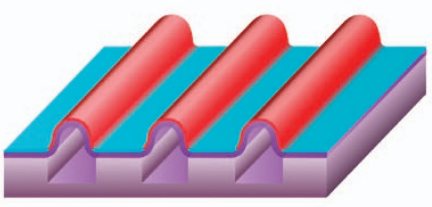

"A" character

b

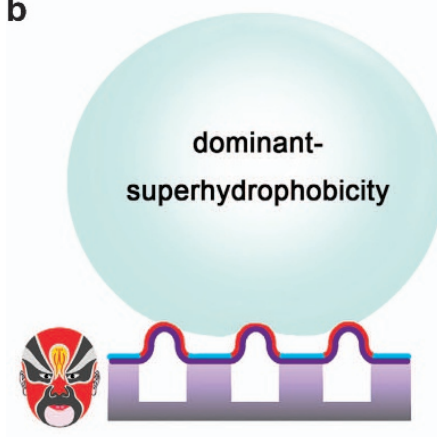

Surface A: red top \& blue bottom

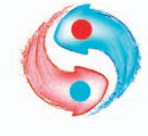

reversible switching

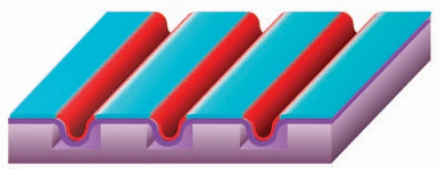

"B" character

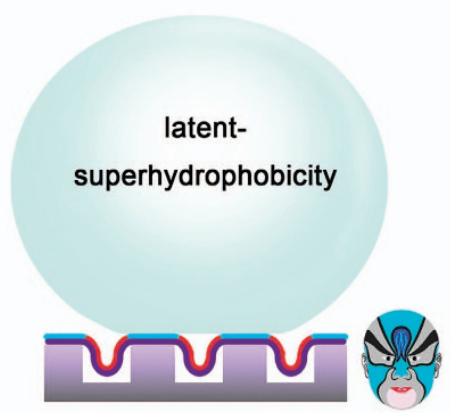

Surface B: blue top \& red bottom

Figure 1 (a) Schematic illustration of the fabrication process of the pneumatic surface. (b) Representation of the concept of a smart surface with dominant and latent superhydrophobicity. The images of opera facial makeup in the insets represent the distinct characters of the surface. 
through hole aligned with the inlet/outlet of the shared air channel. The tubing was tightly inserted into the hole, and the other side was connected to an external pump.

\section{Characterization}

Scanning electron microscopy images were obtained using a JEOL JSM-7500 fieldemission scanning electron microscope (JEOL Ltd, Tokyo, Japan). Wettability

a

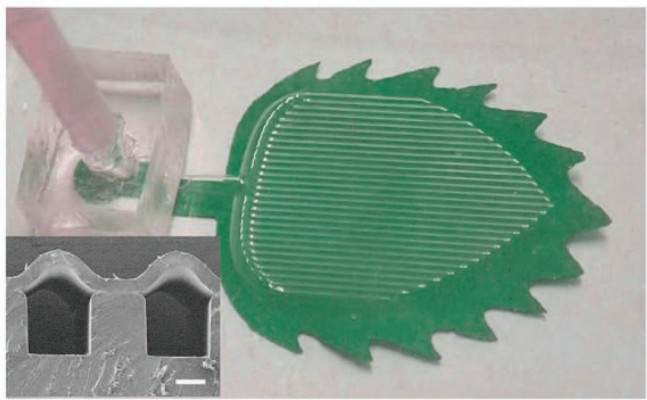

C

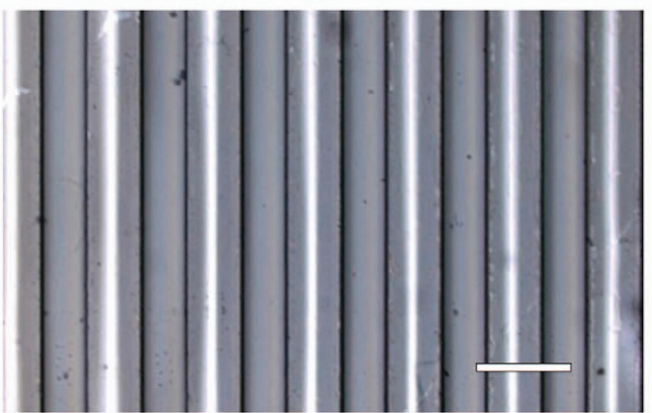

e

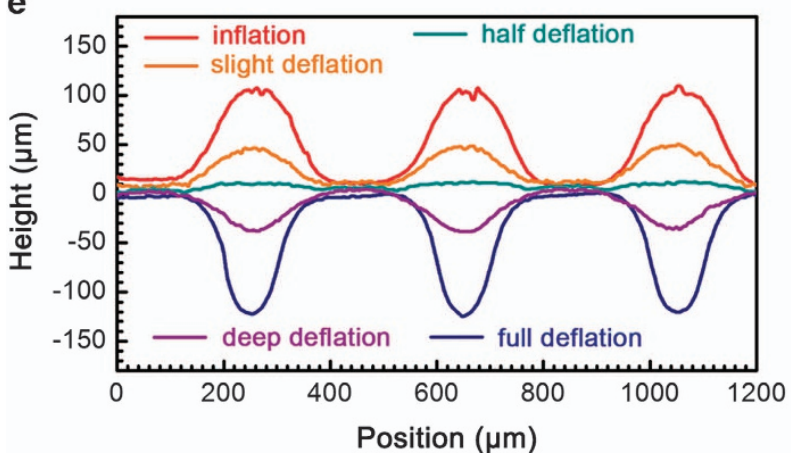

i

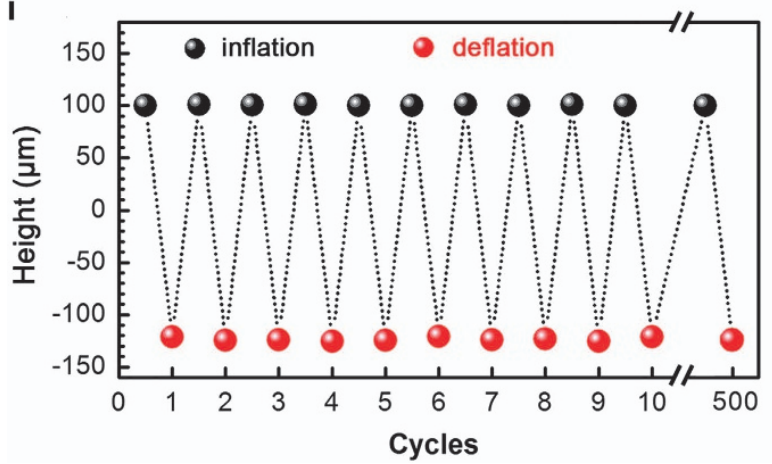

measurement was carried out using a Contact Angle Meter SL200B (Solon Tech., Shanghai, China) at ambient temperature. The contact angles (CAs) and sliding angles (SAs) were measured by the sessile-drop method with a water droplet of $4 \mu$ l. Optical microscopy and confocal laser scanning microscopy images were captured using a LEXT OLS4100 3D measuring laser microscope (Olympus Corporation, Tokyo, Japan).

b

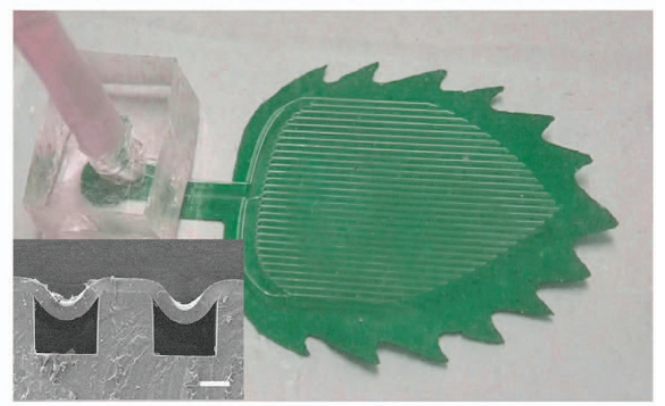

d

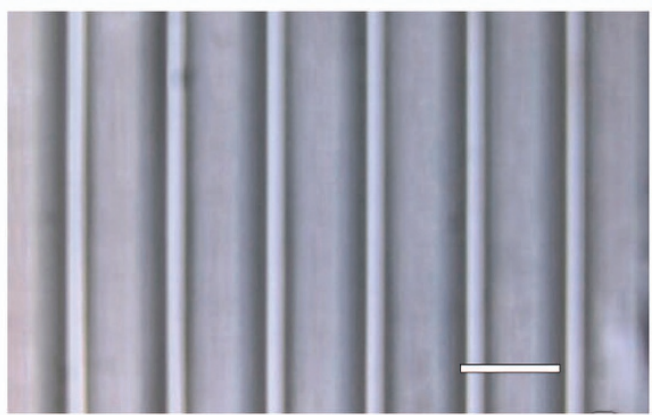

f
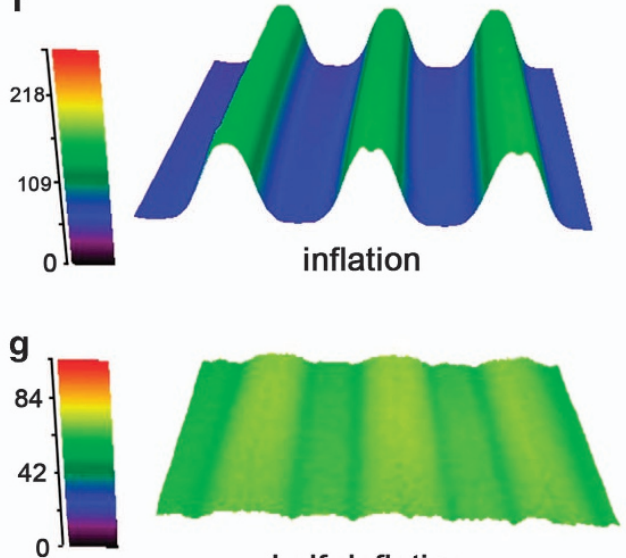

half deflation

h

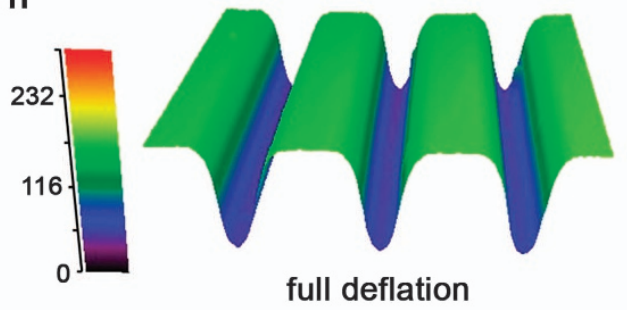

Figure 2 (a, b) Photographs and cross-sectional structures (insets) of the leaf-shaped pneumatic surface in the inflated (a) and deflated (b) states. Scale bars, $100 \mu \mathrm{m}$. (c, d) Optical microscopy (OM) images of the surface in the inflated (c) and deflated (d) states. Scale bars, $400 \mu \mathrm{m}$. (e-h) Height profiles (e) and three-dimensional (3D) images ( $\mathbf{f}-\mathbf{h})$ of the surface during deflating process. (i) Reversible structural deformation. 


\section{RESULTS AND DISCUSSION}

\section{Design principle}

The basic design principle of a smart surface bearing switchable dualsurface properties lies in the construction of a deformable surface that enables controllable orogeny at the microscale. With the rise and fall of the deformation region, a hill could change into a basin. As water droplets are always brought into contact with the protuberant region first, the surface would exhibit distinct dewetting properties as long as the deformation region could be selectively modified with different materials or nanostructures. To achieve this goal, we designed and fabricated a pneumatic micro-air-sac network to regulate the surface orogeny. Considering the flexibility of elastomers and their ease of processing, we chose the commercially available PDMS as the base material that is widely used in lab-on-a-chip systems ${ }^{34}$ and soft actuators. ${ }^{29}$ We prepared the protuberant micro air sacs through a stretching-assisted mismatch-bonding process (Figure 1a). The PDMS substrate with the patterned grooves was pre-stretched and then covalently bonded with a relaxed PDMS slice. Because of the mismatch in strain, micro air sacs with a dome configuration formed after the tension was released. As revealed in the cross-sectional photographs (Supplementary Information, Supplementary Figure S1), the embedded micro air sacs were uniform without local clogging and delamination. The irreversible bonding guaranteed the airtightness and the adjacent insulation of the pneumatic system. Rapid, reversible and largeamplitude topography deformation could be realized by controllable inflation and deflation of the micro air sacs. Importantly, after selective modification of the protuberant micro air sacs or the fixed ground part with functional materials or structures, the pneumatic surface exhibited distinct surface properties. Upon inflation, the structures located over the air sacs (the red region) are entirely 'exposed' to water droplets, revealing 'A' character, whereas upon deflation, these parts are totally 'hidden', and the ground parts (the blue region) are in turn 'exposed', presenting 'B' character. Considering that the inflation and deflation of the micro air sacs would facilitate the selective modification of the top and the ground parts, respectively, any two distinct behaviors could be easily imparted to the pneumatic surface by using different materials or micro-/nanostructures. In this way, the smart surface possesses dual properties, and the distinct surface properties can be switched on demand, similar to the unique face-changing performance in Chinese opera (Figure 1b). Moreover, as the pneumatic operation enables fast and reversible switching of the 'exposed' character on demand, such a smart surface would provide a currently underexploited platform for switchable smart surfaces.

\section{Pneumatic performance}

Based on the abovementioned structures, we could further control the surface orogeny via air pumping. As a proof of concept, we designed this pneumatic surface as a smart leaf with the micro air sacs operating as interconnected leaf veins. As a shared air channel, the petiole was connected to an external air pump. Open to the atmosphere, the device was initially inflated (Figure 2a). Upon deflation, rapid deformation occurred on the surface (Figure $2 \mathrm{~b}$ ). This process was reversible: when pressurized, it could recover the initial state. Figure $2 \mathrm{c}$ and $\mathrm{d}$ shows optical microscopy images of the local details of the inflated and deflated areas, respectively, confirming the surface topological change at the microscale. Because of the rapid transport of air, switching between the inflated and deflated states throughout the surface $(1.1 \mathrm{~cm} \times 2.2 \mathrm{~cm})$ could be completed within a second (Supplementary Information, Supplementary Video S1).

A major advantage of the pneumatic strategy is the ability to dynamically in situ control the surface structure at the microscale. To test the tuning performance, we monitored the responsive morphology in real time using confocal laser scanning microscopy. By controlling the deflation degree, we could modulate the surface configuration continuously. Figure 2e records five typical states observed in this process, displaying gradual changes in amplitude, with maximum values of $100 \mu \mathrm{m}$ at inflation and negative $120 \mu \mathrm{m}$ at full deflation. In particular, we could convert the rough structure (Figure 2f) into a nearly nonstructured flat surface (Figure 2g), as shown in the three-dimensional images. The deflated surface (Figure $2 \mathrm{~h}$ ) assumed a similar configuration to that of the inflated surface (Figure 2f), resembling an inverted reflection. Note that reliability is very important for soft pneumatic devices. In our process, we employed plasma-assisted irreversible bonding to create a robust junction and optimized the thickness of the cover slice to balance the mechanical strength and the bending flexibility. The performance of our pneumatic surface was very stable. After 500 inflation-deflation cycles, the amplitude of the sac remained nearly constant (Figure 2i).

\section{Pneumatic wettability control}

To effectively control wettability under pneumatic actuation, several aspects need to be considered. (1) The cover slice served as a deformable layer; thus, to avoid pneumatic rupture and bending resistance, we selected a layer thickness of $40 \mu \mathrm{m}$ to afford sufficient resilience without losing toughness. (2) To ensure a large amplitude structural deformation, the groove arrays should provide enough space for the deformable layer. (3) To achieve apparent directional wetting performance, the period of the groove array should be comparable to that of natural systems, for instance, rice leaves $(300-400 \mu \mathrm{m}) .^{35}$ Our micro air sacs are designed with large periods and heights (denoted $\mathrm{P}$ and $\mathrm{H}$, respectively, in Figure 3a). To study the influence of structural geometry on surface wettability, we fabricated a series of micro air sacs with different parameters. The period of the structure could be varied from 300 to $500 \mu \mathrm{m}$ by using different SU-8 templates, as shown in the cross-sectional scanning electron microscopy images (Figure $3 \mathrm{~b}$ ). Meanwhile, altering the stretching strain allowed the heights of the structure to be tuned from 40 to $100 \mu \mathrm{m}$ (Figure 3c). To investigate the surface wettability under pneumatic control, we tested the contact angles (denoted $\theta$ ) and sliding angles (denoted $\alpha$ ) as static and dynamic metrics, respectively, in directions perpendicular and parallel to the orientation of the micro air sacs (denoted $\perp$ and $\|$, respectively, in Figure 3a). As typical representatives, we selected two groups of samples for comparison: a $60-\mu \mathrm{m}$-height group with periods ranging from 300 to $500 \mu \mathrm{m}$ and a $400-\mu \mathrm{m}$-period group with heights ranging from 40 to $100 \mu \mathrm{m}$. In addition, we tested the samples under complete deformation for uniformity. In the inflated state, all of the surfaces demonstrated the Wenzel wetting mode. Water droplets adhered to the surfaces upon contact with relatively small $\theta_{\|}$values ranging from $116.9^{\circ}$ to $126.9^{\circ}$. For samples with the same height, the water CAs decreased in both directions as the period increased (Figure 3d). When the period was fixed, the water CAs increased in both directions as the height increased (Figure 3e). These results indicated that a smaller period and a taller height would afford a larger CA, as these parameters provided larger roughness factors according to the Wenzel model. ${ }^{36}$ Moreover, these surfaces showed large gaps between $\theta_{\|}$and $\theta_{\perp}$, ranging from $16.9^{\circ}$ to $24.9^{\circ}$, demonstrating a distinct anisotropy of static wettability resulting from the asymmetrical surface structures of the convex sacs. In the deflated state, the wetting behaviors exhibited similar values and identical tendencies to those in the inflated state, as the deflated surfaces showed the same period and a comparable height to those after inflation (Figure $2 \mathrm{f}$ and $\mathrm{h}$ ). As the micro air sacs could deflect to a flat shape (Figure $2 \mathrm{~g}$ ), the anisotropic wetting mode could be switched to an isotropic one. In this way, we may envisage a 
a

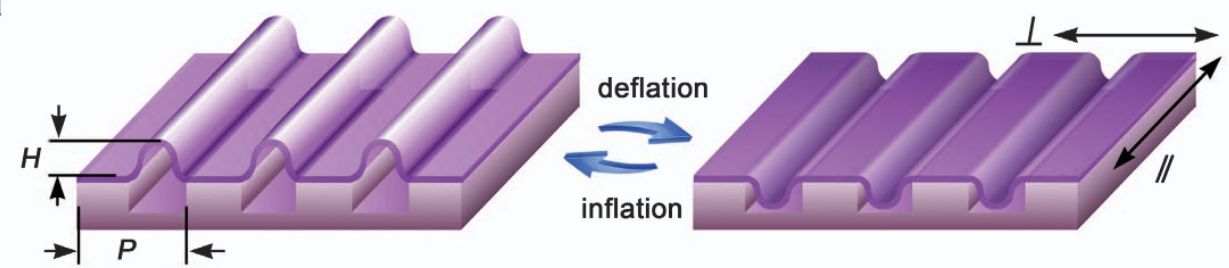

b
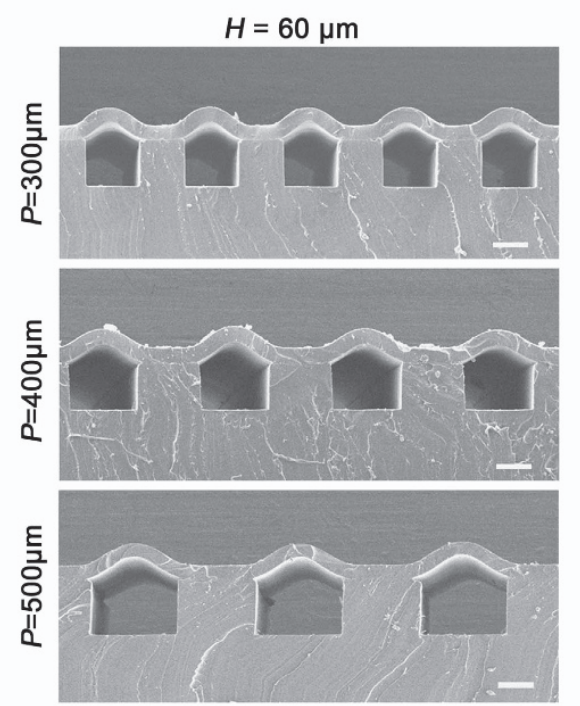

d

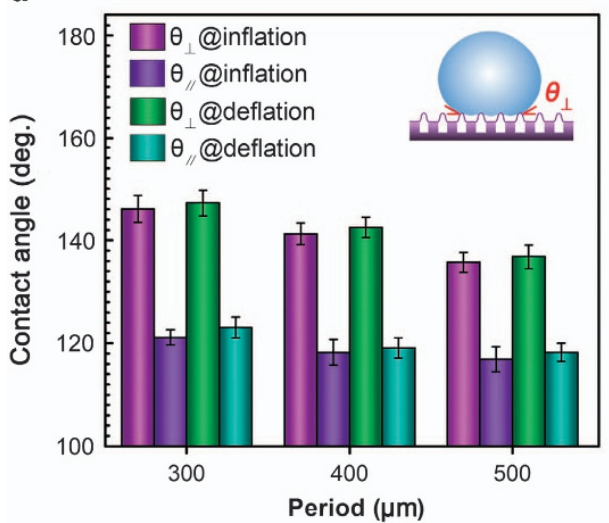

C
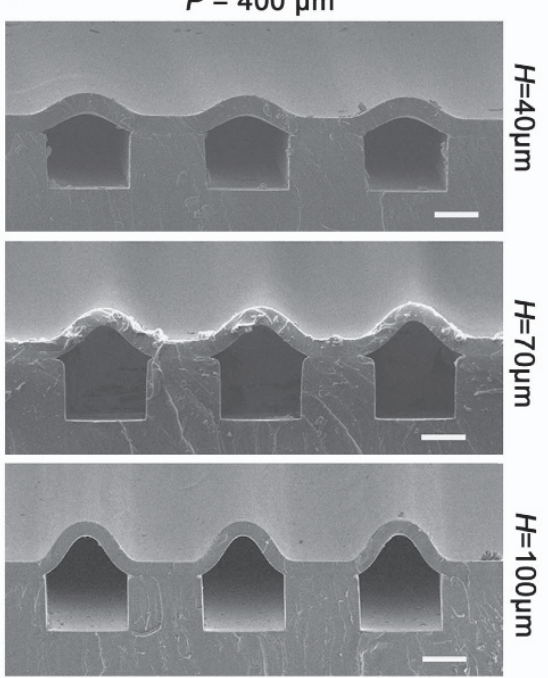

e

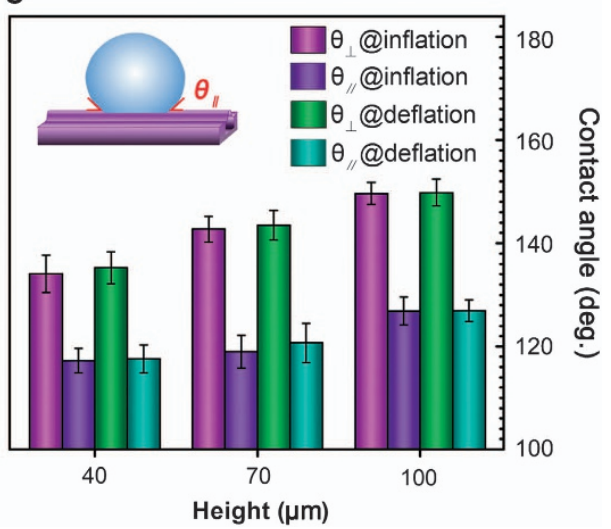

Figure 3 (a) Schematic illustration of the surface topography under pneumatic control. (b, c) Scanning electron microscopy (SEM) images of the 60- $\mu$ mheight group (b) and the 400- $\mu \mathrm{m}$-period group (c) of pneumatic surfaces. Scale bars, $100 \mu \mathrm{m}$. (d, e) Contact angle (CA) tests for the $60-\mu \mathrm{m}$-height group (d) and 400- $\mu \mathrm{m}$-period group (e) of pneumatic surfaces. The insets in (d) and (e) are schematic illustrations of the CAs measured in the perpendicular and parallel directions, respectively.

continuous adjustment of wettability based on the well-controlled structural change (Figure 2e), and this is of great value for smart devices towards adaptive applications. ${ }^{15}$

It has been proved that nanostructures are very important for superhydrophobicity ${ }^{37}$ and dynamic functional materials. ${ }^{38}$ To achieve drastic water repellency, we further modified the surfaces with carbon soot, ${ }^{33}$ as shown in Figure $4 \mathrm{a}$. The resultant surface retained its morphology at the microscale; meanwhile, the entire surface was homogeneously covered with aggregated carbon nanoparticles, yielding hierarchical multiscale structures (Figure $4 \mathrm{~b}-\mathrm{d}$ ). With this method, all the modified surfaces became highly hydrophobic, as droplets resting on them maintained spherical shapes with CAs larger than $150^{\circ}$. In contrast to the unmodified samples, the droplets could roll off the surfaces after a slight disturbance. To further evaluate the dewetting properties, the same two groups of pneumatic surfaces were subjected to SA tests. In the inflated state, for the $60-\mu \mathrm{m}$-height group, $\alpha_{\perp}$ increased as the period increased from 300 to $500 \mu \mathrm{m}$, and the sliding anisotropy, defined as the difference between the SAs in the two directions, also increased (Figure 4e). For the $400-\mu \mathrm{m}$-period group, $\alpha_{\perp}$ and the sliding anisotropy monotonically increased as the height increased (Figure 4f). These results indicated that water droplets preferred to roll off along the sacs, permitting directional control of droplet motion. Thus, dynamic wetting behavior was dependent on the dimensions of sacs as well. For samples with a larger period and a taller height, the sliding anisotropy was more apparent because of the greater energy barrier for water to cross transversally. Because of similar surface structures, the samples in the deflated state showed similar behaviors to those in the inflated state for the two groups. 
a

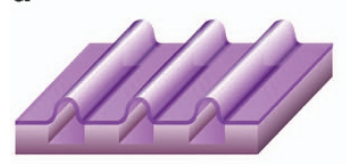

carbon soot coating

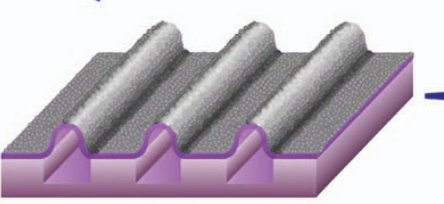

( adhesive tape sticking

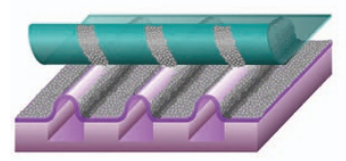

$y$ top soot removal

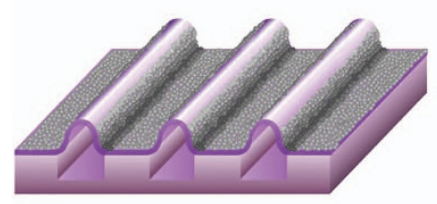

deflation

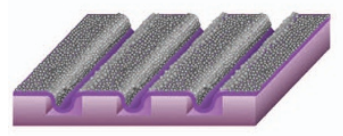

b

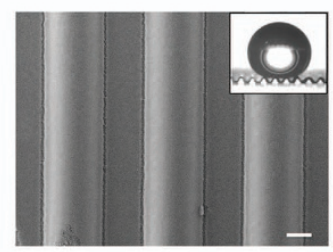

e

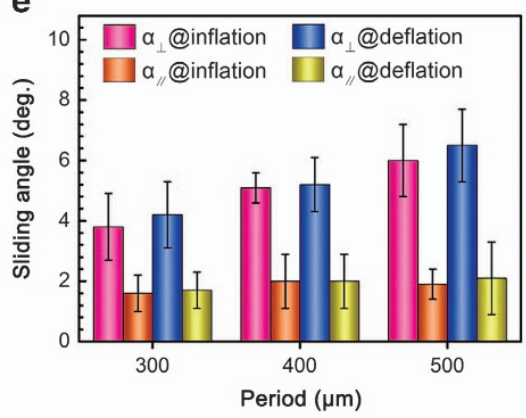

c

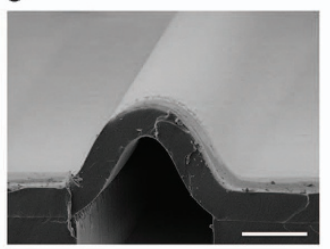

f

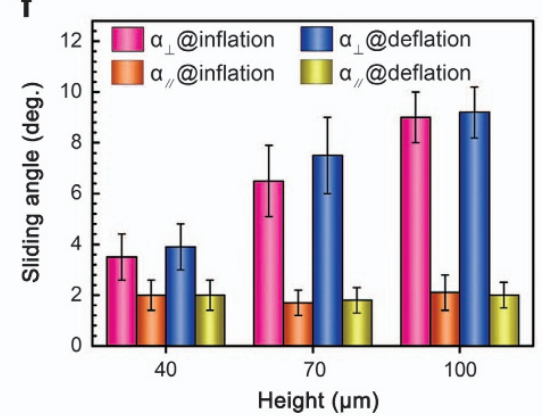

d

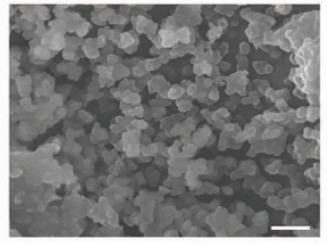

g

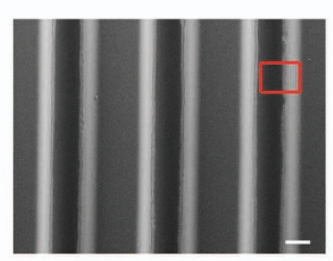

j

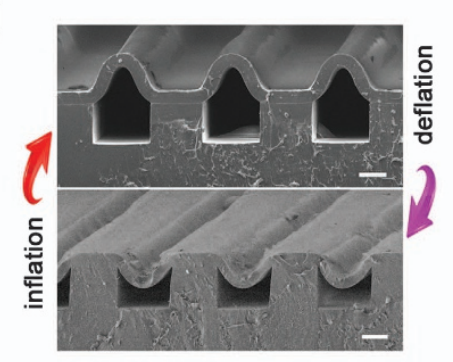

h

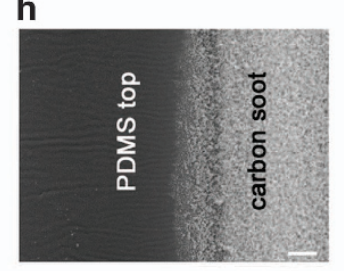

k

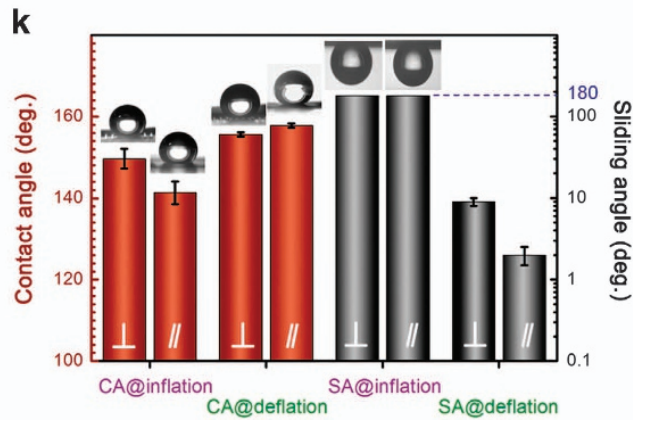

Figure 4 (a) Schematic illustration of the modification process of pneumatic surfaces. (b-d) Scanning electron microscopy (SEM) images of the fully modified surface. The inset shows a photograph of a water droplet on the modified surface. (e, f) Sliding angle (SA) tests for the 60- $\mu \mathrm{m}$-height group (e) and 400- $\mu \mathrm{m}$-period group (f) of modified pneumatic surfaces. ( $\mathbf{g}-\mathbf{i})$ SEM images of the hybrid surface. (j, $\mathbf{k}$ ) SEM images (j) and wetting behaviors (k) of the hybrid surface with 100- $\mu \mathrm{m}$-height and $400-\mu \mathrm{m}$-period micro air sacs under pneumatic control. The insets show photographs of water droplets on the surface in the parallel and perpendicular directions. Scale bars: (b, c, g, i, j) $100 \mu \mathrm{m}$, (d) $500 \mathrm{~nm}$ and (h) $10 \mu \mathrm{m}$.

The analyses and comparisons of both CA and SA tests produced the rule that each pneumatic surface presents similar wetting behaviors in the inflated and deflated states because of the similarity in surface structure and composition. To endow the pneumatic surfaces with distinct personalities, we selectively removed the coating (soot) from the top of the inflated surface using an adhesive tape (Figure 4a), producing uniform hybrid structures with two alternate parts. Scanning electron microscopy images (Figure $4 \mathrm{~g}-\mathrm{i}$ ) show that the naked PDMS region appeared smooth and was clearly distinguished from the rough soot-coated region. In this way, we realized the dynamic tuning of surface structures and materials through pneumatic operation (Figure 4j). Accordingly, tunable wetting behaviors were achieved. We selected the sample with $100-\mu \mathrm{m}$-height and $400-\mu \mathrm{m}$-period sacs as an example, in which two distinct types of wetting phenomena were observed for different pneumatic cases (Figure 4k). The inflated surface showed high CAs $\left(\theta_{\perp}=149.6^{\circ}, \theta_{\|}=141.3^{\circ}\right)$ and a strong affinity for droplets even when the surface was inverted, behaving similar to a rose petal. ${ }^{12}$ In contrast, the deflated surface exhibited high CAs $\left(\theta_{\perp}=155.6^{\circ}, \theta_{\|}=157.8^{\circ}\right)$ but low SAs $\left(\alpha_{\perp}=9^{\circ}, \alpha_{\|}=2^{\circ}\right)$, resembling a lotus leaf. ${ }^{8}$ In this regard, a smart surface that could mimic both the petal effect and the lotus effect was successfully developed. Even though they are two opposite wetting states, rapid and reversible switching between them was achieved by facile inflation and deflation.

\section{Mechanism of wettability switching}

To investigate the underlying mechanism of the wettability transition, especially the contact mode at the complex interface, we observed the water/solid interfaces using optical microscopy. When inflated, the hybrid surface was composed of smooth uncoated PDMS top and rough coated bottom regions (Supplementary Figure S2a, left). Upon contact with water, the bottom region was totally blocked by white clouds (Supplementary Figure S2b, left), indicating the presence of air 
a

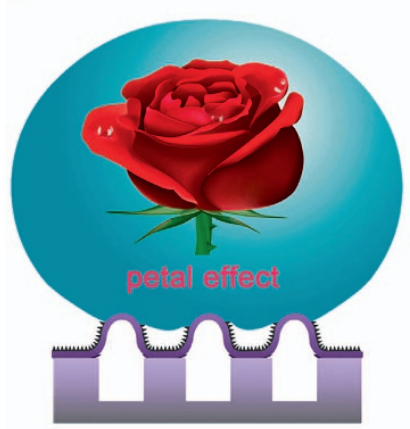

Composite wetting mode

b

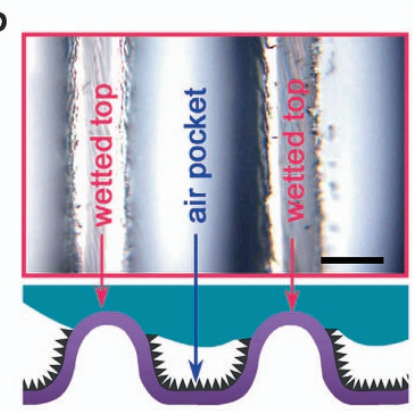

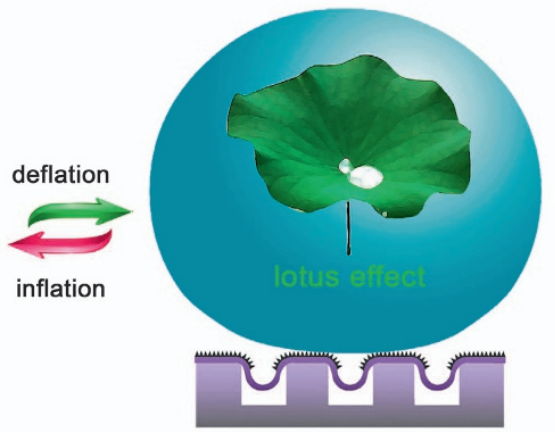

Cassie's wetting mode

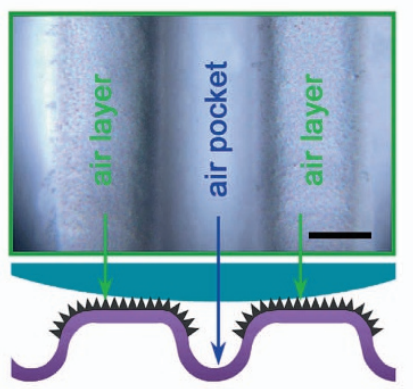

c
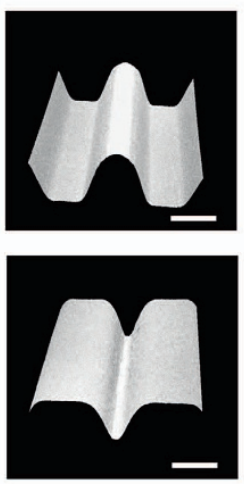

d

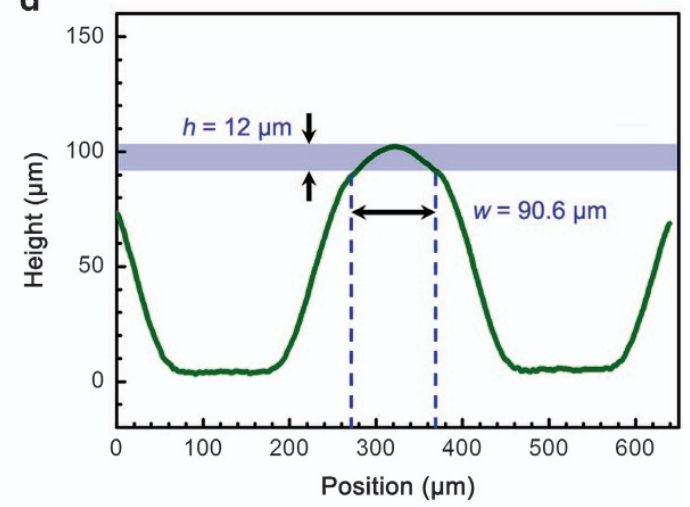

e
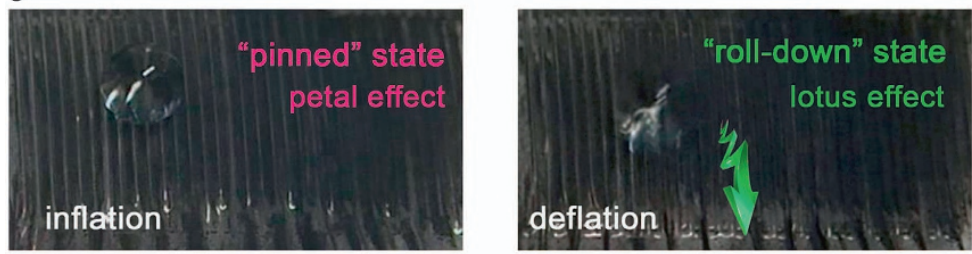

f

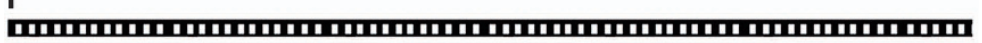

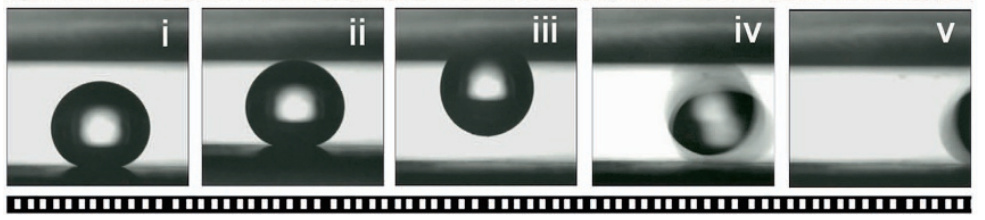

Figure 5 (a, b) Schematic illustration (a) and optical microscopy (OM) images (b) of the water/solid interfaces in the inflated (left) and deflated (right) states. Scale bars, $200 \mu \mathrm{m}$. (c) Three-dimensional (3D) confocal laser scanning microscopy (CLSM) images of the surface in the inflated (top) and deflated (bottom) states. Scale bars, $200 \mu \mathrm{m}$. (d) Height profile of the inflated surface. (e) Photographs of a water droplet stuck on the inflated surface (left) and rolling off the deflated surface (right). (f) Application as a mechanical hand for droplet manipulation. 
pockets that were trapped below the droplet and that covered the entire coated surface. The enlarged local details further revealed that the clouds were constrained right between the borders of the smooth areas (Supplementary Figure S2c, left), causing the water droplet to fully wet the uncoated PDMS but preventing it from infiltrating the bottom region. Based on these results, we proposed a wetting model for the inflated state (Figure $5 \mathrm{a}$ and $\mathrm{b}$, left). According to the CassieBaxter model, ${ }^{39}$ the contact mode in the composite air/liquid/solid system can be described as follows:

$$
\cos \theta_{\mathrm{C}}=f_{\text {sol }} \cos \theta_{\text {sol }}+f_{\text {air }} \cos \theta_{\text {air }}
$$

where $\theta_{\mathrm{C}}$ is the apparent CA in the Cassie model; $\theta_{\text {sol }}$ and $\theta_{\text {air }}$ are the ideal CAs of water on the smooth surface (for PDMS, $\theta_{\text {sol }}=111.0^{\circ}$, Supplementary Figure S3a) and air $\left(\theta_{\text {air }}=180^{\circ}\right)$, respectively; and $f_{\text {sol }}$ and $f_{\text {air }}$ are the fractions of the solid/water interface and the air/water interface $\left(\theta_{\text {sol }}+\theta_{\text {air }}=1\right)$, respectively.

In particular, because of the structural feature, part of our surface (the wetted top area) complied with the Wenzel model ${ }^{36}$ that can be presented as follows:

$$
\cos \theta_{\mathrm{W}}=r \cos \theta
$$

where $\theta_{\mathrm{W}}$ is the apparent CA in the Wenzel model, $\theta$ is the ideal CA of water on the flat surface and $r$ is the surface roughness factor that is defined as the ratio of the actual surface area to the projected area. By modifying the water/solid section of the Cassie equation (Equation 1), the composite contact mode in the inflated state is given as follows:

$$
\cos \theta_{\text {inf }}=r f_{\text {sol }} \cos \theta_{\text {sol }}+f_{\text {sol }}-1
$$

Assuming that the profile of the uncoated part was an arc, we obtained the roughness factor as follows:

$$
r=\frac{2 R}{w} \times \arcsin \frac{w}{2 R}
$$

where $R\left(=\frac{4 h^{2}+w^{2}}{8 h}\right)$ is the radius of the arc and $w$ and $h$ are the width and height of the arc, respectively. The topological and dimensional details of the surface could be determined from the confocal laser scanning microscopy images (Figure $5 \mathrm{c}$ (top) and Figure $5 \mathrm{~d}$ ). Therefore, $\theta_{\text {inf }}$ was calculated as $149.1^{\circ}$, consistent with the measured value $\left(\theta_{\perp}=149.6^{\circ}\right.$, Figure $\left.4 \mathrm{k}\right)$. On the other hand, the fully wetted region provided a seamless liquid/solid contact and strong van der Waals interactions, ${ }^{23}$ explaining why a droplet was attached to the surface even though it was highly hydrophobic. Here, we call this composite state the pinned state (Figure 5e, left).

When the surface was deflated, the smooth PDMS part deformed down toward the bottom, and the top region became a rough plateau (Supplementary Figure S2a, right). To analyze the wetting mechanism, we observed the water/solid interface on the deflated surface. As shown in the optical microscopy images (Supplementary Figure S2b and $c$, right), the surface was thoroughly wrapped by white clouds, including the top and bottom areas. It can be deduced that the droplet was simply sitting on the tips of the rough coating, mainly because it was lifted by air, as shown in the schematic diagram (Figure $5 \mathrm{a}$ and $\mathrm{b}$, right). According to the Cassie equation, the water CA of the deflated surface, $\theta_{\text {def }}$, can be calculated from that of the flat coated $\operatorname{PDMS}\left(\theta_{\text {sol }}{ }^{\prime}=151.2^{\circ}\right.$, Supplementary Figure S3b). Based on the profile of the deflated surface (Figure $5 \mathrm{c}$ (bottom) and Supplementary Figure S3c), we obtained the theoretical value of $\theta_{\text {def }}\left(157.9^{\circ}\right)$ that is comparable to the measured $\theta_{\perp}$ value of $155.6^{\circ}$ (Figure $4 \mathrm{k}$ ). In addition, the trapped air acted as an isolation layer, leading to a significant reduction in contact area and water/solid interaction. When the surface was deflated, the liquid droplet that previously adhered to the inflated surface (Figure 5e, left) could roll off effortlessly (Supplementary Video S2), and this state is called the roll-down state (Figure 5e, right). Moreover, as mentioned above, the droplet directionally moved along the micro air sacs.

To obtain further insight into the switching performance, we characterized the change in both the surface structure (Supplementary Figure S4a and Supplementary Video S3) and water CA (Supplementary Figure S4b and c and Supplementary Video S4) during the pneumatic switching. Notably, the surface structure clearly changes during the switching between inflation and deflation. Accordingly, the CAs also change slightly. Despite the droplet on the surface showing excellent water repellency with high CAs that are always larger than $150^{\circ}$, relative larger CAs appear in the deflated states (Supplementary Figure S4c). Nevertheless, the amplitude of the CA change is small, that is, within $5^{\circ}$. In addition to the static CA changes, we also evaluated the CA hysteresis along two directions during the pneumatic switching (Supplementary Figure S5). CA hysteresis, defined as the difference between the advancing $\mathrm{CA}\left(\theta_{\mathrm{a}}\right)$ and receding $\mathrm{CA}\left(\theta_{\mathrm{r}}\right)$, evidently changes when the surface is largely tilted $\left(20^{\circ}\right)$, indicating a dynamic alteration in the adhesive property during the state transition. The droplet was firmly stuck to the inflated surface but immediately rolled down the tilted surface upon deflation. This dynamic process was carefully studied in directions perpendicular (Supplementary Figure S5a and c and Supplementary Video S5) and parallel (Supplementary Figure S5b and d and Supplementary Video S6) to the orientation of the air sacs. With an increasing degree of deflation, the CA hysteresis of the tilted surface sharply decreased (Supplementary Figure S5e) in both directions, showing pneumatically controlled contrastive wettability. Moreover, CAs in the parallel direction presented a smaller hysteresis because of the structural asymmetry.

\section{Manipulation of a water droplet}

It is well known that the manipulation of small amounts of liquid, especially for an expensive and valuable sample, is an important issue in the areas of biomedicine and microfluidics. Utilizing the unique switching between the pinned and roll-down ultrahydrophobic states, we presented an on-off manipulator for water droplet transportation. Figure $5 f$ shows the working process (Supplementary Video S7). Here, a superhydrophobic glass was used to support the water droplet ready for transfer. Upon contact with the inflated surface, the water droplet could be immediately captured by the surface because of the 'petal effect'. The droplet adhered to the surface could be subsequently transferred to the desired location, where the droplet could be released within a second by simply deflating the micro air sacs and thus switching to the lotus state. In this way, droplets can be transported using a single smart surface. Notably, as a droplet on a superhydrophobic surface has a tiny contact area, cross-contamination could be effectively avoided. Moreover, the biocompatible PDMS surface, actuated in a green and rapid pneumatic manner, would provide a safe and efficient means of liquid manipulation.

\section{CONCLUSION}

In summary, a pneumatic smart surface that possesses switchable dual properties has been successfully developed based on a common elastomer using a stretching-assisted mismatch-bonding process. We first fabricated periodically distributed micro air sacs with a protuberant configuration, permitting rapid and large-amplitude topographical deformation under pneumatic control. Because of its sufficient robustness and flexibility, the surface could undergo hundreds of inflation/deflation cycles without damage. Then, nanoscale roughness was introduced to the entire surface that contributes to the formation of hierarchical structure and superhydrophobicity. Under pneumatic control, the protuberant micro air sacs could be gradually deflated from a 
convex shape to a flat and even concave shape. In this way, free switching between anisotropic/isotropic wetting behaviors can be realized. Furthermore, fast and reversible switching between a 'petal' effect and a 'lotus' effect was achieved by selectively removing the nanoscale structures on the top of the micro air sacs. Based on the transition between the two opposite wetting states, the smart surface was implemented as a manipulator of droplet transfer. Our results provide an alternative protocol for the rational design and fabrication of smart surfaces with tunable wettability and introduce new possibilities for expanding this field beyond conventional stimuli-driven behaviors (for example, $\mathrm{pH}$, ultraviolet light, temperature and solvent). In fact, the micro air sacs can be designed in a desired distribution with adjustable dimensions, and the modified structures and materials can be extensively varied. Therefore, this pneumatic strategy enables us to build a general intelligent system capable of switching between all kinds of wetting behaviors, and it would provide a platform based on a novel concept for the development of intelligent surfaces with tunable topology-correlated properties.

\section{CONFLICT OF INTEREST}

The authors declare no conflict of interest.

\section{ACKNOWLEDGEMENTS}

We acknowledge the National Key Research and Development Program of China and National Natural Science Foundation of China under Grants \#2017YFB1104300, \#61522503, \#61775078, \#61590930 and \#61605055 for support.

\section{PUBLISHER'S NOTE}

Springer Nature remains neutral with regard to jurisdictional claims in published maps and institutional affiliations.

1 Tian, Y., Su, B. \& Jiang, L. Interfacial material system exhibiting superwettability. Adv Mater. 26, 6872-6897 (2014).

2 Neinhuis, C. \& Barthlott, W. Characterization and distribution of water-repellent, selfcleaning plant surfaces. Ann. Bot. 79, 667-677 (1997).

3 Gao, X. F. \& Jiang, L. Water-repellent legs of water striders. Nature 432, 36-36 (2004).

4 Parker, A. R. \& Lawrence, C. R. Water capture by a desert beetle. Nature 414, 33-34 (2001).

5 Sanchez, C., Arribart, H. \& Guille, M. M. G. Biomimetism and bioinspiration as tools for the design of innovative materials and systems. Nat. Mater. 4, 277-288 (2005).

6 Wang, S. T., Liu, K. S., Yao, X. \& Jiang, L. Bioinspired surfaces with superwettability: new insight on theory, design, and applications. Chem. Rev. 115, 8230-8293 (2015).

7 Sun, T. L., Qing, G. Y., Su, B. L. \& Jiang, L. Functional biointerface materials inspired from nature. Chem. Soc. Rev. 40, 2909-2921 (2011).

8 Barthlott, W. \& Neinhuis, C. Purity of the sacred lotus, or escape from contamination in biological surfaces. Planta 202, 1-8 (1997).

9 Zhang, F. Z., Zhao, L. L., Chen, H. Y., Xu, S. L., Evans, D. G. \& Duan, X. Corrosion resistance of superhydrophobic layered double hydroxide films on aluminum. Angew. Chem. Int. Ed. 47, 2466-2469 (2008).

10 Lee, C. \& Kim, C. J. Underwater restoration and retention of gases on superhydrophobic surfaces for drag reduction. Phys. Rev. Lett. 106, 014502 (2011).

11 Mishchenko, L., Hatton, B., Bahadur, V., Taylor, J. A., Krupenkin, T. \& Aizenberg, J. Design of ice-free nanostructured surfaces based on repulsion of impacting water droplets. ACS Nano 4, 7699-7707 (2010).

12 Feng, L., Zhang, Y. A., Xi, J. M., Zhu, Y., Wang, N., Xia, F. \& Jiang, L. Petal effect: a superhydrophobic state with high adhesive force. Langmuir 24, 4114-4119 (2008).

13 Hong, X., Gao, X. F. \& Jiang, L. Application of superhydrophobic surface with high adhesive force in no lost transport of superparamagnetic microdroplet. J. Am. Chem. Soc. 129, 1478-1479 (2007).

14 Su, B., Wang, S. T., Ma, J., Wu, Y. C., Chen, X., Song, Y. L. \& Jiang, L. Elaborate positioning of nanowire arrays contributed by highly adhesive superhydrophobic pillarStructured substrates. Adv. Mater. 24, 559-564 (2012).

15 Yao, X., Hu, Y. H., Grinthal, A., Wong, T.-S., Mahadevan, L. \& Aizenberg, J. Adaptive fluid-infused porous films with tunable transparency and wettability. Nat. Mater. 12, 529-534 (2013).
16 Zang, J. F., Ryu, S., Pugno, N., Wang, Q. M., Tu, Q., Buehler, M. J. \& Zhao, X. H. Multifunctionality and control of the crumpling and unfolding of large-area graphene. Nat. Mater. 12, 321-325 (2013).

17 Huber, D. L., Manginell, R. P., Samara, M. A., Kim, B. I. \& Bunker, B. C. Programmed adsorption and release of proteins in a microfluidic device. Science 301, 352-354 (2003).

18 Wong, W. S. Y., Gutruf, P., Sriram, S., Bhaskaran, M., Wang, Z. K. \& Tricoli, A. Strain engineering of wave-like nanofibers for dynamically switchable adhesive/repulsive surfaces. Adv. Funct. Mater. 26, 399-407 (2016).

19 Lv, T., Cheng, Z., Zhang, D., Zhang, E., Zhao, Q., Liu, Y. \& Jiang, L. Superhydrophobic surface with shape memory micro/nanostructure and its application in rewritable chip for droplet storage. ACS Nano 10, 9379-9386 (2016).

20 Zheng, W. F., Zhang, W. \& Jiang, X. Y. Precise control of cell adhesion by combination of surface chemistry and soft lithography. Adv. Healthcare Mater. 2, 95-108 (2013).

21 Mertens, S. F. L., Hemmi, A., Muff, S., Groning, O., De Feyter, S., Osterwalder, J. \& Greber, T. Switching stiction and adhesion of a liquid on a solid. Nature 534, 676-679 (2016).

22 Xin, B. W. \& Hao, J. C. Reversibly switchable wettability. Chem. Soc. Rev. 39, 769-782 (2010).

23 Liu, M. J. \& Jiang, L. Switchable adhesion on liquid/solid interfaces. Adv. Funct. Mater. 20, 3753-3764 (2010)

24 Wang, S. T., Feng, X. J., Yao, J. N. \& Jiang, L. Controlling wettability and photochromism in a dual-responsive tungsten oxide film. Angew. Chem. Int. Ed. 45, 1264-1267 (2006).

25 Yu, X., Wang, Z. Q., Jiang, Y. G., Shi, F. \& Zhang, X. Reversible pH-responsive surface: from superhydrophobicity to superhydrophilicity. Adv. Mater. 17, 1289-1293 (2005).

26 Minko, S., Muller, M., Motornov, M., Nitschke, M., Grundke, K. \& Stamm, M. Two-level structured self-adaptive surfaces with reversibly tunable properties. J. Am. Chem. Soc. 125, 3896-3900 (2003).

27 Wu, D., Wu, S.-Z., Chen, Q.-D., Zhang, Y.-L., Yao, J., Yao, X., Niu, L.-G., Wang, J.-N., Jiang, L. \& Sun, H.-B. Curvature-driven reversible in situ switching between pinned and roll-down superhydrophobic states for water droplet transportation. Adv. Mater. 23, 545-549 (2011).

28 Wigglesworth, V. B. A further function of the air sacs in some insects. Nature 198, 106-106 (1963).

29 Mosadegh, B., Polygerinos, P., Keplinger, C., Wennstedt, S., Shepherd, R. F., Gupta, U., Shim, J., Bertoldi, K., Walsh, C. J. \& Whitesides, G. M. Pneumatic networks for soft robotics that actuate rapidly. Adv. Funct. Mater. 24, 2163-2170 (2014).

30 Wehner, M., Truby, R. L., Fitzgerald, D. J., Mosadegh, B., Whitesides, G. M., Jewis, J. A. \& Wood, R. J. An integrated design and fabrication strategy for entirely soft, autonomous robots. Nature 536, 451-455 (2016).

31 Zhang, W., Zappe, H. \& Seifert, A. Wafer-scale fabricated thermo-pneumatically tunable microlenses. Light Sci. Appl. 3, e145 (2014).

32 Cooksey, G. A. \& Atencia, J. Pneumatic valves in folded 2D and 3D fluidic devices made from plastic films and tapes. Lab Chip 14, 1665-1668 (2014).

33 Deng, X., Mammen, L., Butt, H. J. \& Vollmer, D. Candle soot as a template for a transparent robust superamphiphobic coating. Science 335, 67-70 (2012).

34 Llobera, A., Juvert, J., Gonzalez-Fernandez, A., Ibarlucea, B., Carregal-Romero, E. Buttgenbach, S. \& Fernandez-Sanchez, C. Biofunctionalized all-polymer photonic lab on a chip with integrated solid-state light emitter. Light Sci. Appl. 4, e271 (2015).

35 Wu, D., Wang, J.-N., Wu, S.-Z., Chen, Q.-D., Zhao, S., Zhang, H., Sun, H.-B. \& Jiang, L. Three-level biomimetic rice-leaf surfaces with controllable anisotropic sliding. Adv. Funct. Mater. 21, 2927-2932 (2011).

36 Wenzel, R. N. Resistance of solid surfaces to wetting by water. Ind. Eng. Chem. 28 988-994 (1936).

37 Liu, K. S. \& Jiang, L. Bio-inspired design of multiscale structures for function integration. Nano Today 6, 155-175 (2011).

38 Ariga, K., Li, J. B., Fei, J. B., Ji, Q. M. \& Hill, J. P. Nanoarchitectonics for dynamic functional materials from atomic-/molecular-level manipulation to macroscopic action. Adv. Mater. 28, 1251-1286 (2016).

39 Cassie, A. B. D. \& Baxter, S. Wettability of porous surfaces. Trans. Faraday Soc. 40 546-550 (1944).

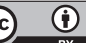

This work is licensed under a Creative Commons Attribution 4.0 International License. The images or other third party material in this article are included in the article's Creative Commons license, unless indicated otherwise in the credit line; if the material is not included under the Creative Commons license, users will need to obtain permission from the license holder to reproduce the material. To view a copy of this license, visit http:// creativecommons.org/licenses/by/4.0/

(C) The Author(s) 2018

Supplementary Information accompanies the paper on the NPG Asia Materials website (http://www.nature.com/am) 